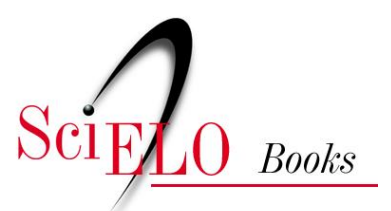

\title{
La escuela como proyecto de expansión de la ciudadanía (1900- 1960) una mirada desde la ribera del río Bobonaza
}

\author{
María Antonia Manresa Axisa
}

\section{SciELO Books / SciELO Livros / SciELO Libros}

MANRESA AXISA, M.A. La escuela como proyecto de expansión de la ciudadanía (1900-1960): una mirada desde la ribera del río Bobonaza. In: JUNCOSA BLASCO, J., and GARZÓN VERA, B., eds. Misiones, pueblos indígenas y la conformación de la Región Amazónica: actores, tensiones y debates actuales [online]. Quito: Editorial Abya-Yala, 2019, pp. 41-55. ISBN: 978-9978-10-494-1. https://doi.org/10.7476/9789978104941.0004.

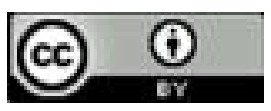

All the contents of this work, except where otherwise noted, is licensed under a Creative Commons Attribution 4.0 International license.

Todo o conteúdo deste trabalho, exceto quando houver ressalva, é publicado sob a licença Creative Commons Atribição 4.0.

Todo el contenido de esta obra, excepto donde se indique lo contrario, está bajo licencia de la licencia Creative Commons Reconocimento 4.0. 


\title{
La escuela como proyecto de expansión de la ciudadanía (1900-1960): una mirada desde la ribera del río Bobonaza ${ }^{1}$
}

\author{
M. Antonia Manresa Axisa ${ }^{2}$ \\ antonia.manresa@uasb.edu.ec
}

\section{Introducción}

En este artículo exploro el surgimiento de la escuela en la provincia de Pastaza durante el periodo de la primera mitad del siglo XX, enfocado principalmente en las comunidades establecidas en la ribera del río Bobonaza.

Empleo métodos etnográficos recogiendo historias de vida de las personas mayores de la comunidad y realizo análisis de archivos históricos. Analizo el surgimiento de las primeras escuelas religiosas de principio de 1900 y el surgimiento de escuelas fiscales a mediados de siglo. Muestro cómo las primeras escuelas surgen como parte del proyecto evangelizador de la misión dominicana y contrasto con el surgimiento de escuelas fiscales en la ribera de los años 40 . 
Argumento que la expansión de la educación pública independiente de la autoridad encargada sostiene como eje central el concepto de conversión evangelizadora frente a la población indígena. Propongo que el 'educar' a la población indígena es conceptualizado como proceso de 'conversión' hacia sujeto civil. Sugiero que la expansión y función de la escuela durante el siglo XX en esta zona está ligada al reconocimiento relativo de ciudadanía del sujeto a ser 'educado'. En otras palabras, la noción de sujeto depende de quién o quiénes pueden ser para el Estado reconocidos como ciudadanos y quienes solo al ser 'educados', pueden potencialmente convertirse y adquirir el estatus de sujeto completo. Concluyo que la expansión de la educación se enmarca en una categorización racial de la población y que existe una continuidad histórica entre el proyecto civilizatorio civil y la de evangelización en torno a la escuela como espacio de instrucción formal.

\section{Contexto histórico}

El proceso de colonización de la Amazonía ecuatoriana inicia con las primeras incursiones llevadas a cabo en 1576 (García, 1999). Para 1636 la corona española con el objetivo de 'civilizar' a los 'salvajes' estableció la misión jesuítica de Maynas (García, 1999), cuya jurisdicción se extendía a la mayor parte de lo que hoy en día representa gran parte de la región amazónica de los países de América del Sur.

El río Bobonaza se encontraba en el corazón de esta designación $y$, por ende, los grupos étnicos que habitaban esta zona fueron incursionados por expediciones misionales para su evangelización mediante el establecimiento de reducciones cristianas desde el siglo XVII. La misión de Canelos, como parte de esta gran jurisdicción jesuita, fue mayormente dominada por la presencia de misioneros dominicanos durante la época colonial. La fundación oficial de la misión de Canelos al parecer es algo controversial, el Padre Pierre señala la fecha para 1581 (Pierre, 1988) aun cuando la fecha comúnmente nombrada es 1624 (Vargas,1934). Desde una perspectiva antropológica, el proceso de 
asentamiento que llevan a cabo los misioneros implica la reconformación de grupos étnicos principalmente a consecuencia de la reducción poblacional a causa de grandes epidemias de viruela y otras enfermedades introducidas desde Europa. Se calcula que la población de la zona se redujo hasta en un $80 \%$ entre el siglo XVII y XVIII (Taylor, 2007). Esta forma de agrupación arbitraria y diversa se puede evidenciar en las narrativas de los padres misioneros. Por ejemplo, el Padre Pierre recogiendo los relatos de los misioneros en las "Relaciones de Indias, 1881" describe el ingreso de los cuatro misioneros dominicanos de 1581 a la región para evangelizar a la población que encontraban y crear reducciones cristianas de la siguiente forma:

Dios no tardó en recompensar [a los Padres] su celo y bendecir sus trabajos. Cinco familias de Gaes o Gayes [Zaparos], que habitaban la ribera derecha del Pastaza, fronteriza a la planicie de Barrancas, se presentaron al Padre Amaya e imploraron la gracia del bautismo. Después de instruidos, recibieron del Padre las aguas regeneradoras de este sacramento, siendo esta tribu el germen de la cristiandad de Canelos...El Padre Quintana, apenas supo este acontecimiento... No tardó en dar con la reducida tribu de los Inmundas, a la cual, catequizando, consiguió convertir a la fe cristiana. Por su parte el Padre Ochoa conquistó pacíficamente a los Hualingas...fusionando luego estos con los Gayes y fundando sobre la orilla izquierda del Pastaza la primera población cristiana...con el nombre de Coninche. Por ese mismo tiempo, el Padre Rosero, presentándose a la cabeza de la tribu de los Santes o Santis, trayendo además los pocos sobrevivientes de la tribu de los Inmundas, azotada y disminuida por las viruelas, y engroso, con este nuevo contingente el número de pobladores de Coninche. Todos, de común acuerdo, decidieron en llamarse los Canelos, porque, según rezan las crónicas, había muchos árboles de canela en este sito. Tal, la circunstancia que influyo para considerar al Padre Rosero como el fundador de Canelos. (Pierre, 1988, p. 124)

En este relato se menciona la disminución poblacional a causa de las epidemias y se describe la forma de agrupar a diversos grupos étnicos. Es curioso cómo el relato no da cuenta 
de ningún tipo de resistencia o conflicto, expresando una visión harmónica y consenso, lo cual es poco probable, ya que el mismo Padre Pierre cuenta cómo este primer asentamiento se tuvo que reubicar cuatro veces, "por desviamientos, ataques de jibaros y abandono” (Pierre, 1988, p. 124).

Es importante puntualizar que, hasta finales del siglo XIX, lo que existen son incursiones misionales que fueron de mayor o menor intensidad dependiendo de las posibilidades de las misiones en distintos periodos, sin embargo, no se consigue sostener 'centros' realmente estables. Para 1775 los archivos de los misioneros indican que Canelos solo contaba con 19 hombres y para 1789 no indican ningún número de habitantes en Canelos (Vargas, 1934). En general los relatos de los misioneros muestran su frustración frente a que de una incursión a otra las reducciones establecidas desaparecen (Vargas, 1934). Esto ocurre ya por múltiples causas entre ellas el abandono voluntario ya que las poblaciones y grupos étnicos no respondían a una lógica de asentamiento permanente, siendo grupos relativamente nómadas en territorios amplios. Otra causa es la devastación poblacional a causa de enfermedades y luchas territoriales entre grupos étnicos. Además, existe un proceso de la migración interna desde las zonas altas hacia las zonas más bajas para escapar de los diezmos que se insertan y conforman parte de estos grupos generando mayor diversidad y diferencias en patrones de asentamiento.

Los misioneros dominicanos nombraron los grupos étnicos de la misión de Canelos como 'Canelenses'. Para inicios de 1800 se reconocen varios asentamientos relativamente permanentes en la rivera del Bobonaza que los misioneros han nombrado pueblo de: Canelos, Pakayaku, Sarayaku y Junjiri (ahora Montalvo). A la reconformación étnica de la Rivera de Bobonaza, Whitten (1976) los describe como pertenecientes a la cultura de los Canelos Quichua que incluye no solo a los grupos de la ribera del Bobonaza hasta Canelos, sino que se extiende al norte hasta los ríos Villano y Curaray y al este hasta Chambira y al sur hasta la cabezera del río Copataza (Whitten, 1976, p. 14).

Los padres jesuitas están presentes en el sector de Canelos de forma intermitente, en 1886 establecen la Prefectura 
Apostólica de Canelos y Macas en lo que es hoy la comunidad

de Canelos. Con la expulsión de la misión jesuita de la región del Napo en 1896, retoman los padres dominicanos ahora de forma permanente estableciendo su residencia en Canelos con tres padres misioneros.

\section{Las primeras escuelas}

Hasta mediados de 1900, el pueblo de Canelos es el centro político de esta zona por la presencia de la sede Apostólica de la misión dominicana y una población de alrededor de 200 personas. El Padre Magallí menciona que para 1892 se había construido en Canelos una casa misional para los tres padres misioneros, una escuela y también residía el comisario de policía y cuatro soldados. Por ende, la primera escuela que se nombra en los documentos históricos es esta de Canelos y su función se explica en el siguiente extracto:

Instalados los misioneros en una cabaña, guardaban el horario convencional; su plan de evangelización, sencillo y práctico: La educación de los niños, recoger los más posibles, de siete y ocho años, comprar a los infieles los hijos que abandonan, dar instrucción a todos y casar a los jóvenes... (García 1999, p. 275)

Este extracto muestra la relación directa de la educación con la visión evangelizadora de los Padres. Sin embargo, es importante entender que estas primeras escuelas funcionaban como orfanatos, en otras palabras, más que escuelas donde los niños de las comunidades asisten, son internados compuestos por un número relativamente pequeño de niños indígenas. Lo que sí se menciona en relación con instrucción formal son pequeños intentos de formación básica en alfabetización, matemáticas e higiene como complemento a las clases de catequesis. Esto se describe, en una carta escrita por el Padre Apostólico de Canelos en 1927 explicando cómo se realizan estas clases a los niños de las comunidades de la Rivera durante las 'reuniones':

Todas las tardes al toque de la campana han acudido...en número más o menos de ochenta, los niños del Catecismo. 
Por el intervalo de una media hora han recibido pequeñas nociones de cultura, se les ha instruido en cuenta numérica y en el conocimiento de las letras y aún se les ha adiestrado en pequeños movimientos de gimnasia higiénica. Hay niños perspicaces que pronto han aprovechado de las instrucciones dadas...

Triste es decirlo, pero la verdad se impone; en el tiempo que vagan en las purinas olvidan mucho de lo que aprendieron del Misionero, de tal suerte que una reunión a otra el Adelanto es poco o nada. Mientras esta arraigada y pésima costumbre de las purinas no desaparezca, ningún progreso de civilización será efectivo en estos pueblos. (Oriente Dominicano, 1927, pp. 15-16)

Las 'reuniones' representan las visitas periódicas de los misioneros a los pueblos de la zona donde reúnen a la gente para realizar la misa, los bautizos, casamientos, catequesis y demás actividades evangelizadoras. Según los relatos en el Oriente Dominicano, estas visitas se realizan una vez al mes durante esta época con estadías de entre varios días a una semana.

Del extracto de arriba lo que podemos entender es que la instrucción formal a los niños de los pueblos asentados en la rivera del Bobonaza era una tarea bastante limitada y que genera frustración por parte de los padres misioneros. Es evidente la visión culturalmente jerárquica de los padres en este momento en donde desconocen las costumbres de los pueblos y ven la instrucción formal como un proceso para civilizar a estas poblaciones. De esta forma se puede interpretar que la educación formal tanto como la evangelización están dirigidas a 'convertir' a los niños en sujetos civilizados.

Los documentos indican que la primera escuela de Puyo se funda en 1914, con cinco niños 'blancos' y cinco niños 'indios' (Gobierno provincial de Pastaza, 2015). Puyo no existe hasta su fundación en 1899 , se convierte en capital de provincia en 1909, sin embargo, es más bien un ideal para crear un centro urbano que una realidad en estos momentos. Lo descrito de viajeros sobre Puyo a principios de 1900 es de estar conformada por "menos de una docena de familias" (Hurtado, 1988). Las comunidades de la rivera de Bobonaza son mucho más grandes, 
con poblaciones de entre 200 a 500 miembros. Mera se funda en 1924, a unos $25 \mathrm{~km}$ de Puyo como el primer asentamiento de 'blanco-colonos' en la región con unas doce familias (Hurtado, 1988). Es así como a principios de 1900 Puyo y Mera representan los inicios de lo que se convertiría en una rápida expansión poblacional y proyecto urbanizador para esta región amazónica. La proyección era "hacer de Puyo el centro de comunicación vial con los principales pueblos de esta región" (Oriente Dominicano, 1936, p. 39). Para finales de los años 20 además del orfanato en Canelos, los misioneros indican sostener “...escuelas gratuitas en Canelos, Puyo y Mera. Estos dos últimos pueblos cuentan ya con instalación de luz y de teléfonos" (Oriente Dominicano 1935, p. 44). Es significativo que son los pueblos de Puyo y Mera que cuentan con luz y servicios de comunicación.

Según los datos poblacionales recopilados por los misioneros estos dos pueblos son los que contienen mayor población 'blanca' nombrada así por parte de los misioneros (Puyo $100 \mathrm{y}$ Mera 350). Esto representa la primera ola de migración interna promovida por políticas de colonización que se oficializo con la declaración de tierras baldías de los años 1937. Es posible identificar como para 1936 la provisión escolar sigue el mismo patrono que los otros servicios concentrado principalmente en los pueblos de Mera y Puyo:

\begin{tabular}{|l|c|c|}
\hline \multirow{2}{*}{ Pueblos } & \multicolumn{2}{|c|}{ Provisión Escolar 1936 } \\
\cline { 2 - 3 } & Misional & Fiscal \\
\hline Puyo & 1 & 1 \\
\hline Mera & 1 & \\
\hline Canelos & 1 & \\
\hline Pacayacu & & \\
\hline Pumamaki & & 1 \\
\hline Montalvo & 3 & 1 \\
\hline Total & & 1 \\
\hline
\end{tabular}

En los años 30, la provisión escolar era de cuatro escuelas, dos en Mera, y una en Puyo y Canelos que corresponden a las zonas de mayor influencia de la misión y de mayor concen- 
tración de población 'blanco-colono'. Es significativo además que Mera con la mayor población 'blanco-colono' es el único pueblo que cuenta dos escuelas, una misional y la otra "mixta del gobierno" (Oriente Dominicano, 1936, p. 39). En las otras comunidades de la Rivera la escuela como una institución del Estado, ya sea fiscal o misional para la enseñanza intencional de estudiantes con personal específico dirigida a esta tarea, no existen hasta los años 40. Esto implica que las primeras escuelas se centran en los centros de expansión urbana y de mayor influencia de los misioneros dominicanos.

\section{Expansión de la provisión escolar}

El crecimiento población de la región a mediados de siglo XX se dispara. Para 1946 Puyo cuenta con una población de alrededor de 1600 personas, mucho mayor que la de las otras comunidades. Si consideramos que a finales de los años 20 Puyo era un pequeño asentamiento, este incremente en el crecimiento se explica a consecuencia de la intensificación de migración y colonización territorial de la Amazonía. El siguiente gráfico demuestra el cambio poblacional de las comunidades de la zona en el transcurso de 1930 a 1946:

Gráfico 1. Cambios demográficos de los principales pueblos cristianos entre 1930 a 1946

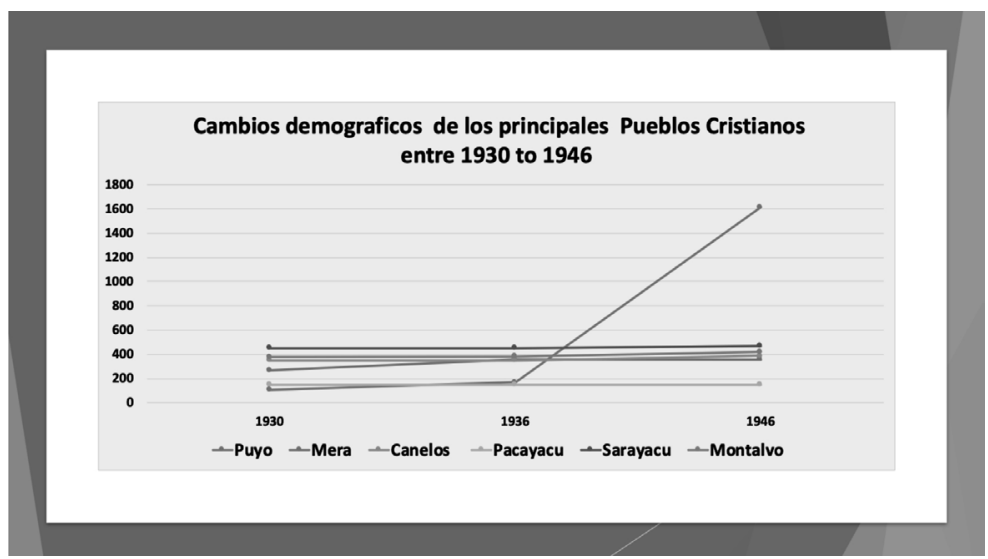


Esta explosión poblacional de Puyo implica cambios demográficos para la zona en general. Esto se puede evidenciar en relación con una categorización racial establecida en este periodo en el siguiente gráfico:

Gráfico 2. Cambios demográficos en relación a una categoría racial entre el periodo 1930-1946

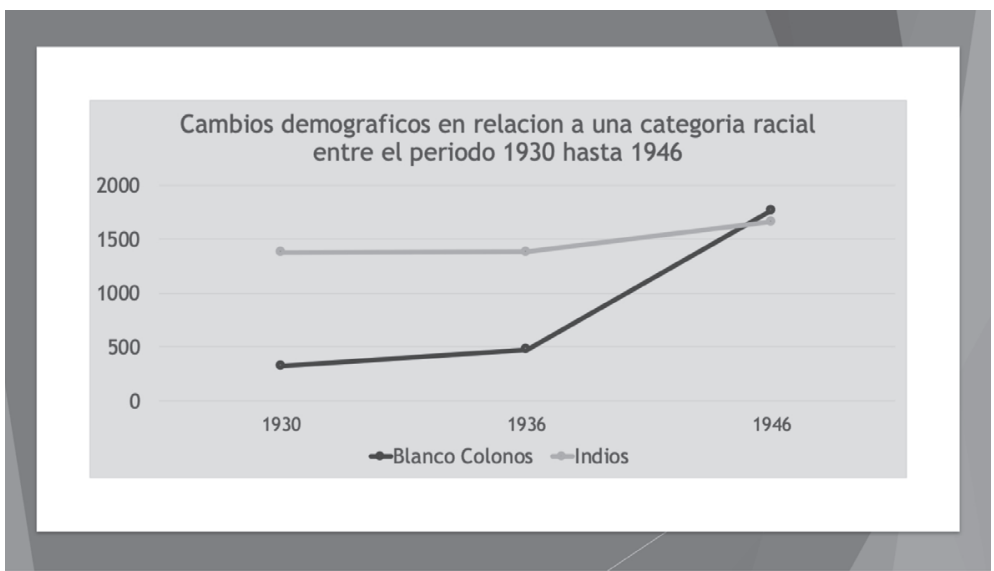

Fuente: Recopilación de datos del Oriente Dominicano 1930-1946, Manresa, 2018.

Lo que muestran estos gráficos es cómo los pueblos de la rivera se mantienen más o menos estables en torno a su población durante este periodo. En Mera se da un regular y leve crecimiento poblacional mientras que en Puyo el crecimiento poblacional se dispara a partir de 1936 . Como resultado, la población migrante para 1946 supera la población indígena históricamente asentada en esta zona. La categorización de los misioneros en términos generales muestra por lo tanto un ligero crecimiento de la población de 'indios' y un crecimiento muy rápido a partir de 1936 de la población 'blanco-colonos' que supera en números totales la población indígena de la zona para 1946. Esto además implica que, en el periodo de la mitad del siglo XX, las poblaciones en relación con una categoría racial se 
concentran en distintas zonas; la población de 'blanco-colonos' se asienta en los centros de expansión urbana y la población de 'indios' en los pueblos tradicionales de la rivera.

La provisión escolar para 1946 también se expande de forma dramática como muestra la siguiente tabla:

Tabla 1.

\begin{tabular}{|c|c|c|c|c|c|c|c|}
\hline & & & & \multicolumn{2}{|c|}{ Escuelas } & \multicolumn{2}{|c|}{ Alumnos } \\
\hline & 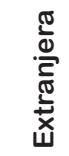 & $\begin{array}{l}0 \\
0 \\
0 \\
\frac{C}{0}\end{array}$ & 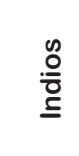 & 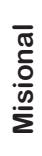 & $\begin{array}{l}\overline{\mathscr{J}} \\
\text { if }\end{array}$ & 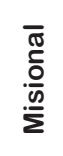 & $\begin{array}{l}\overline{\mathscr{J}} \\
\text { iq }\end{array}$ \\
\hline Puyo & 25 & 1300 & 315 & 1 & 1 & 85 & 60 \\
\hline Mera & 20 & 349 & 30 & 1 & 1 & 34 & 28 \\
\hline Canelos & & 45 & 514 & 1 & 1 & 26 & 3 \\
\hline Pacayacu & 1 & 9 & 190 & & & & \\
\hline Pumamaki & & 19 & 352 & & 1 & & 8 \\
\hline Montalvo & 1 & 50 & 263 & & 1 & & 12 \\
\hline Total & 47 & 1772 & 1664 & 3 & 5 & 145 & 138 \\
\hline
\end{tabular}

Fuente: Revista El Oriente Dominicano, 1946 reproducida de la tabla "Estadísticas Generales de Prefectura Apostólica de Canelos”, p. 88.

La tabla muestra el censo poblacional en términos de la categorización racial establecida durante este periodo: 'blancos, indios y extranjeros'. Esta clasificación racial de la población se puede entender relativa a una percepción racial de Estado que identifica una población 'civilizada', en contraste con otra. En este sentido la población migrante 'blanco-colono' es percibida como civilizada frente a los aún no civilizados 'indios'. La categorización racial 'blanco/indio' en este contexto equivale al concepto dualista; 'civilizado/no civilizado'. Este análisis sugiere la expansión de la provisión de escuelas que se establecen en los años 40 responden al incremento poblacional categorizado como 'blancos'. El término blanco no necesariamente corres- 
ponde a un color de piel sino a reconocimiento diferenciado

de estatus como de personas 'civilizadas' frente a la población local denominada 'indios'.

En la tabla se indica además el tipo de escuela, fiscal o misional y el número de estudiantes en estas escuelas. Es significativo que la expansión de la provisión educativa en este periodo es representada por la expansión de escuelas fiscales, que además de estar presentes en Mera y Puyo se extienden a los pueblos de la Ribera; Canelos, Sarayacu y Montalvo.

En números totales, el número de estudiantes que atienden a escuelas fiscales y misionales es parecido; 145 para las escuelas misionales y 138 para las escuelas fiscales. Sin embargo, los 138 estudiantes de las escuelas fiscales están mayormente distribuidas en Puyo y Mera, para las tres escuelas restantes; Canelos, Sarayacu y Montalvo solo atienden 23 estudiantes a las escuelas fiscales.

La pregunta que surge es: ¿Por qué aparecen escuelas fiscales en estas comunidades consideradas tanto 'remotas' y principalmente de 'indios', para servir un número tan reducido de estudiantes?

Revisando la tabla se puede ver que las escuelas fiscales están presentes donde hay población denominada 'blanco', solo Pacayacu con el número menor de blancos (9) no cuenta con una escuela fiscal. Por lo tanto ¿están estas escuelas fiscales sirviendo a esta población de 'blancos'? Esto podría ser una respuesta lógica, sin embargo, llama la atención los datos de Canelos ya que a diferencia de las otras comunidades de la Ribera Canelos contaba ya con una escuela dirigida por los misioneros. La tabla muestra que esta escuela misional cuenta con 26 estudiantes y la escuela fiscal solo cuenta con tres estudiantes. Por tanto, la pregunta se convierte no solo en ¿para quién sirven estas escuelas, sino qué función tienen en este momento histórico en particular? Para contestar esta pregunta es necesario adentrarse a los detalles con los testimonios de las personas que vivieron este periodo. Para esto me refiero a los testimonios de algunos mayores de la comunidad de Sarayacu que cuentan que la primera escuela aparece entre 1941-1942 como una escuelita fiscal. 
Doña Elsa cumplía 82 años, es una de las hijas de la primera familia "mezclada" de Sarayacu. Ella se acuerda haber asistido a esta primera escuela a la edad de 6 o 7 años ubicada a la subida de la plaza central que consistía en un cuarto con techo de paja. Doña Elsa me cuenta que "en esos tiempos no es como ahora, antes las familias vivían más esparcidas y solo las familias de colonos vivían agrupadas", me indica que al otro lado del río se estableció un destacamento militar (testimonio de Doña Elsa, en Manresa, 2018, p. 35).

Cuando le pregunté quiénes asistían a la escuela, Elsa me comentó que eran mayormente los hijos de las familias de los militares. Según Elsa los niños (hombres) de las familias indígenas asistían de forma irregular y no ponían mucha atención siendo castigados por el maestro. En su opinión, las familias indígenas no valoraban la educación llevándose a sus hijos a las purinas por largos periodos (testimonio de Don Sergio en Manresa, 2018, p. 36). Don Sergio, mayor a Doña Elsa por quizás unos diez años me explicó que él como adolescente asistía a la escuela de rato en rato y solo para aprender las letras y español. Me confiesa que en verdad él no aprendió mucho en la escuela solo cuando su tío como primer profesor indígena de la zona fue el docente por unos años "algo hacía entender, traduciendo las palabras" (testimonio de Don Sergio en Manresa, 2018, p. 40). El primer profesor indígena, que menciona Don Sergio fue miembro de la comunidad y formado por la misión dominicana como interno para hacerse cura. Según testimonios de los mayores de la comunidad al no tener las 'aptitudes' para hacerse cura por algunos vicios, no es aceptado para esto, por lo que los padres le incentivan para entrar a la docencia. Sin embargo, algunos de los mayores me explican que a los indígenas no se les permitía entrar a ser padres y los padres misioneros encontraban escusas. Según Don Sergio con su tío aprendió lo básico, pero realmente aprendió a hablar el español ‘bien' cuando salió a trabajar en la costa.

Según estos testimonios y los relatos que expresan los padres dominicanos en la revista Oriente Dominicano que se man- 
tiene hasta mediados de 1950, durante la primera parte del siglo $\mathrm{XX}$, las familias indígenas no tenían mayor interés en una escuela en su territorio y no usaban estas escuelas de forma regular. Estas escuelas servían, como explica Doña Elsa, a hijos de familias colonas y militares. La conclusión, por tanto, de la expansión de la provisión escolar de escuelas fiscales en esta zona es que sirven en estos momentos principalmente a la población denominada como 'blanca', en otras palabras, a la población que el Estado reconoce como sujetos y ciudadanos. Sin embargo, es algo curioso que a inicios de los 40 el Estado prácticamente de la noche a la mañana asignara estos recursos y esfuerzos para establecer escuelas fiscales en estas poblaciones para un número tan pequeño de estudiantes especialmente como ya se ha mencionado, considerando los datos de Canelos ya que cabe la pregunta; ¿por qué se establecen escuelas fiscales y no misionales?

La respuesta está, no en la función educativa que pretende cumplir la escuela (educar a los hijos e hijas de los ciudadanos que el Estado reconoce como tal), sino en el valor simbólico que representa la escuela, específicamente la escuela fiscal para este momento histórico en particular. Las escuelas fiscales de la rivera del Bobonaza aparecen en las comunidades donde a principio de los años 40 también se establecen destacamentos militares con sus familias. Es a principios de estos mismos años cuando surge el enfrentamiento territorial entre Ecuador y Perú, con el resultado de la firma del Protocolo de Río de Janeiro en 1941. Por tanto, estas escuelas fiscales responden a la necesidad del Estado de mostrar presencia no solo militar sino principalmente civil para legitimar soberanía territorial. Es relevante que las escuelas fiscales de estas comunidades de la Rivera para mediados de los años 1950 desaparecen. La interpretación es que al reducirse el conflicto limítrofe territorial el Estado no considera la necesidad de mantener escuelas fiscales en estas comunidades de población principalmente indígena y se le retorna la responsabilidad de 'educar' a estas poblaciones a la iglesia.

Según testimonios en la comunidad de Sarayacu, se regresa a una situación parecida a la anterior donde los padres siguen 
visitando y dan instrucciones, pero además surge la influencia de la iglesia protestante. Durante un periodo, a mediados de los años 50, existe una escuelita que informalmente da clases dirigida por una familia protestante evangélica de un matrimonio entre un miembro de la comunidad y una mujer colona.

En conclusión, la escuela representa un símbolo civilizatorio con sus matices particulares. A principios del siglo XX los padres dominicanos son los encargados por parte del Estado de 'educar y convertir' a las nuevas generaciones de indios en sujetos 'completos' y potenciales ciudadanos. Pero, se puede entender que esto es más bien una visión ilusoria que no se completa ya que no existe mayor interés en la instrucción formal por parte de la comunidad local. Para los años 40 la provisión de escuelas está principalmente dirigida a servir a los nuevos ciudadanos colonos pioneros que sí son reconocidos por parte del Estado. La expansión escolar, por ende, forma parte de la expansión de ciudadanía en relación con el proyecto político nacional de Estado moderno y monocultural. En relación con la rivera del Bobonaza, la escuela fiscal cumple la función de presencia del Estado de forma simbólica, frente a la precaria soberanía territorial de este periodo.

Según los testimonios de los mayores en mi trabajo etnográfico la escuela fue abiertamente rechazada por la organización política y familias indígenas o puntalmente funcional para 'aprender el español y las letras' (Manresa, 2018). No es hasta principios de los años 60 cuando en el caso de Sarayacu, la organización pide el establecimiento de una escuela permanente con el apoyo de los padres dominicanos. Esto coincide con un periodo de alto conflicto y amenaza de pérdida total de relativa autonomía territorial a consecuencia de la expansión de la reforma agraria que inicia en los años 30 y se consolida con la primera ley de reforma agraria y de colonización de 1964. Durante la reforma agraria solo las personas reconocidas como ciudadanos pueden acceder a títulos de las tierras. Como he argumentado, la población indígena local durante este periodo no es reconocida como sujeto completo requiriendo ser educa- 
do como proceso de civilización. Puntalmente no es hasta 1979

en que la Constitución reconoce a las personas no alfabetizadas como ciudadanos y, por ende, sujetos políticos.

Resumiendo, la visión de la mayor parte del siglo XX es de educar a la población indígena local como proceso de conversión hacia sujetos civilizados que recae en la función evangelizadora de la misión. Las escuelas fiscales de los años 40, por lo tanto, no necesitan cumplir la función de educar a niños indígenas ya que sirven mayormente como un acto simbólico de presencia civil sirviendo a los niños y niñas de la población que sí es reconocida como ciudadanos 'completos' por parte del Estado. No es hasta los años 60 cuando la población local siente la necesidad de tener acceso a la escuela para servir como reconocimiento directo por parte del Estado de que también son ciudadanos.

\section{Bibliografía}

García, L. (1999). Historia de las misiones en la Amazonía ecuatoriana, Segunda Edición. Quito: Abya-Yala.

Gobierno Provincial de Pastaza (2015). Pastaza reseña histórica. Recuperado de: https://bit.ly/2XTo3H1 (12 de abril 2015).

Hurtado, H. (1988). Pastaza: Antecedentes históricos, cronología, bibliografía. Quito,: Centro de Investigaciones CIUDAD.

Manresa, M. A. (2018) Intercultural Bilingual Education in Ecuador as a site of negotiation and struggle over difference: A case study of an Amazonian Kichwa school system (Tesis doctoral). Newcastle University, UK.

Misión Dominicana de Ecuador (1926-1946). Revista El Oriente Dominicano.

Pierre, F. (1988). Viaje de exploración al Oriente ecuatoriano 18871888. Quito: Abya-Yala.

Taylor, A. C. (2007). Sick of history: Contrasting regimes of historicity in the Upper Amazon. Time and memory in indigenous Amazonia: anthropological perspectives, 133-168.

Vargas (1934) Monografía sintética de la Misión Dominicana de Canelos y guía del cantón Pastaza. Recuperado de: https://bit. ly/30uAczA (15 de julio 2014). 